Coupled thermoelasticity in a composite half-space

Parnell, William J

2005

MIMS EPrint: 2006.135

Manchester Institute for Mathematical Sciences

School of Mathematics

The University of Manchester

\footnotetext{
Reports available from: http://eprints.maths.manchester.ac.uk/

And by contacting: The MIMS Secretary

School of Mathematics

The University of Manchester

Manchester, M13 9PL, UK
} 


\title{
Coupled Thermoelasticity in a Composite Half-Space.
}

\author{
W.J. Parnell \\ School of Mathematics, University of Manchester, \\ Oxford Road, Manchester, M13 9PL.
}

June 1, 2006

\begin{abstract}
The problem of fully coupled thermoelasticity in a composite half-space is considered where the composite has variations in its physical properties in one direction only. The resulting one dimensional problem thus depends on the so called microscale of the composite. Homogenization of the fully coupled theory provides the leading order system of coupled equations (independent of the microscale) together with the effective physical properties of the thermoelastic medium. In particular the effective coupling parameter $\delta_{*}$ is found and it is shown to exhibit rather interesting properties; for a range of volume fractions in two-phase composites it is shown that $\delta_{*}$ lies below the corresponding coupling parameter for a homogeneous material made up of either phase.

Transient boundary value problems of the homogenized system are then solved and compared with the classical problem of a homogeneous half space. The magnitude of resulting discontinuities in field variables and their derivatives are found and their dependence on the effective coupling parameter is exhibited.
\end{abstract}

Keywords: Coupled thermoelasticity, homogenization, effective moduli.

\section{Introduction}

The fully coupled theory of linear thermoelasticity incorporates the effect of strain on temperature and vice versa. We can often make simplifications to this theory which lead to a partial decoupling of the system but there are situations when it is necessary to retain the fully coupled theory of thermoelasticity, the details of which may be found in Boley and Weiner [5].

In this article we are interested in solving transient boundary value problems which require the use of the fully coupled theory in media which are inhomogeneous. By this we mean that their material properties vary from point to point on a lengthscale $l$ and for composites this is usually much smaller than the characteristic lengthscale $L$ of the body itself and of the lengthscale of variation $\eta$ in field variables such as displacement and temperature. This is termed a separation of scales within the medium $(l \ll \eta$ and $l \ll L)$. We consider the one dimensional version of the theory, for an inhomogeneous medium which is restricted to move only in the $x$ direction and which is uniform in directions perpendicular to the $x$ axis, an example of which is depicted in figure 1 . The body has periodic microstructure (with period $l$ ) along the $x$ axis, so that it can be characterized by a periodic cell (see figure 2 for an example). On scaling the lengthscales within the body with respect to $\eta$, we can appeal to the 


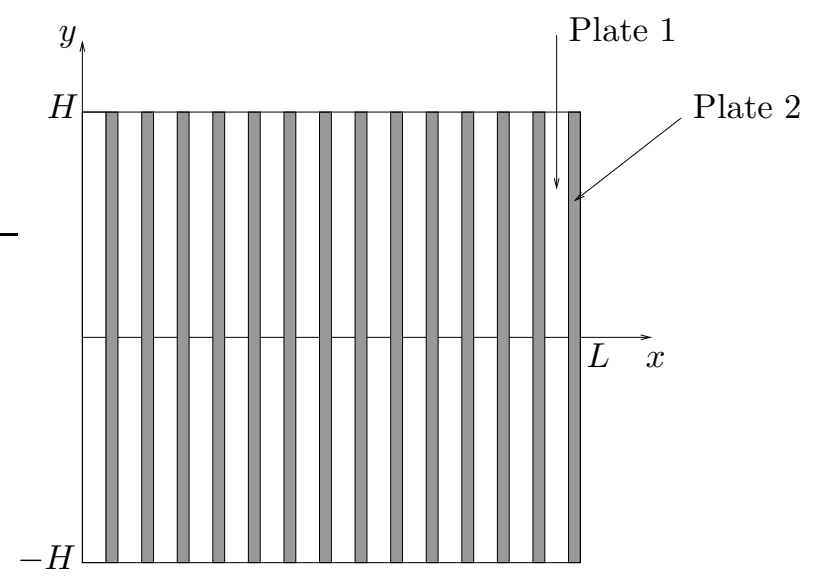

Figure 1: Figure showing the type of composite under discussion in this article.

separation of scales within the medium to define the small parameter $\epsilon=l / \eta$ and the homogenization of the composite then corresponds to the limit $\epsilon \rightarrow 0$. Since we are interested in a subset of problems for a thermoelastic, composite half-space in $x \geq 0$, we consider the limit $L / \eta, H / \eta \rightarrow \infty$, with reference to figure 1 . Clearly a half-space does not exist in practice and we really mean that $L / \eta$ and $H / \eta$ are large enough so that "end effects" can be neglected and this will be reasonable for small enough times, the regime in which we are interested here.

In this context, the modified heat equation is given by

$$
\begin{aligned}
\frac{\partial}{\partial x}\left(k\left(\frac{x}{\epsilon}\right) \frac{\partial \Theta_{\epsilon}}{\partial x}(x, t)\right)- & \rho\left(\frac{x}{\epsilon}\right) c_{v}\left(\frac{x}{\epsilon}\right) \frac{\partial \Theta_{\epsilon}}{\partial t}(x, t) \\
& =\left(3 \lambda\left(\frac{x}{\epsilon}\right)+2 \mu\left(\frac{x}{\epsilon}\right)\right) \alpha\left(\frac{x}{\epsilon}\right) \Theta_{0} \frac{\partial^{2} u_{\epsilon}}{\partial x \partial t}(x, t) .
\end{aligned}
$$

The subscript $\epsilon$ denotes that the fields vary on the lengthscale $\epsilon$ and we note that the material properties are functions of the microscale $x / \epsilon$. The temperature and $x$ component of displacement in the medium are denoted by $\Theta_{\epsilon}$ and $u_{\epsilon}$ respectively, whilst $k, c_{v}$ and $\alpha$ are the thermal conductivity, specific heat (at constant volume), and thermal expansion coefficients. The elastic moduli of the medium are denoted by $\lambda$ and $\mu$, and $\rho$ denotes its mass density. Finally $\Theta_{0}$ is the temperature at which the medium is stress free and we assume that this parameter is independent of $x$. We see in (1.1) that rates of change of the dilatation in time cause variations in temperature and thus mechanical energy is converted into heat. Care must be taken with differentiation in (1.1) if, as is the case in figure 1 , the material property $k(x / \epsilon)$ is piecewise constant. Homogenization results in the integration of such discontinuous quantities however and therefore we do not encounter any problems.

The thermal stress $\sigma_{\epsilon}$ in the medium, allowing for thermal expansion, is 
given by

$$
\begin{aligned}
\sigma_{\epsilon}(x, t)=\left(\lambda\left(\frac{x}{\epsilon}\right)+2 \mu\right. & \left.\left.\frac{x}{\epsilon}\right)\right) e_{\epsilon}(x, t) \\
& -\left(3 \lambda\left(\frac{x}{\epsilon}\right)+2 \mu\left(\frac{x}{\epsilon}\right)\right) \alpha\left(\frac{x}{\epsilon}\right)\left(\Theta_{\epsilon}(x, t)-\Theta_{0}\right)
\end{aligned}
$$

where the linear strain tensor $e_{\epsilon}(x, t)$ is defined as

$$
e_{\epsilon}(x, t)=\frac{\partial u_{\epsilon}}{\partial x}
$$

The equation of motion of the one dimensional, inhomogeneous medium is therefore given by

$$
\begin{aligned}
\frac{\partial}{\partial x}\left\{\left(\lambda\left(\frac{x}{\epsilon}\right)+\right.\right. & \left.\left.2 \mu\left(\frac{x}{\epsilon}\right)\right) \frac{\partial u_{\epsilon}}{\partial x}(x, t)\right\}=\rho\left(\frac{x}{\epsilon}\right) \frac{\partial^{2} u_{\epsilon}}{\partial t^{2}}(x, t) \\
& +\frac{\partial}{\partial x}\left\{\left(3 \lambda\left(\frac{x}{\epsilon}\right)+2 \mu\left(\frac{x}{\epsilon}\right)\right) \alpha\left(\frac{x}{\epsilon}\right)\left(\Theta_{\epsilon}(x, t)-\Theta_{0}\right)\right\} .
\end{aligned}
$$

Equations (1.1) and (1.4) constitute the coupled system in which we are interested. For a homogeneous material, we can neglect the coupling in the modified heat equation provided that (in one dimension) [5]

$$
\frac{\partial e_{\epsilon}}{\partial t} /\left(\alpha \frac{\partial \Theta_{\epsilon}}{\partial t}\right) \ll\left(\frac{3 \lambda+2 \mu}{\lambda+2 \mu}\right) \frac{1}{\delta}
$$

where

$$
\delta=\frac{(3 \lambda+2 \mu)^{2} \alpha^{2} \Theta_{0}}{(\lambda+2 \mu) \rho c_{v}}
$$

is the thermoelastic coupling parameter, indicating the strength of the coupling due to the dilatation term in the modified heat equation (1.1). Since $\delta \ll 1$ in most metals, with reference to (1.5) we may neglect the coupling provided that strain rates and temperature rates are of the same order.

The homogeneous thermoelastic half-space problem was first considered by Danilovskaya [8] who considered the problem when the surface of the half-space is suddenly heated. Boley and Tolins [4] discussed the problem further by analysing the mathematically convenient strain boundary condition and Achenbach [1] described a way to incorporate the coupling approximately by replacing the dilatation term in equation (1.1) with a delta function.

More recently, the problem of an inhomogeneous, periodic, layered medium constituting a thermoelastic half-space has been considered by Bacynski [2] who used a so called "refined averaged model" in order to homogenize the material, find its effective properties and solve transient boundary value problems. It is not clear to the present author how this method is an improvement of the more conventional homogenization procedure used here and outlined by Bakhvalov and Panasenko [3] for example. In particular the fact that the microscale dependence appears to be guessed in [2] is a little disturbing, particularly since effective properties are then stated as functions of this microshape function. Furthermore the work of Bacynski [2] neglects the coupling referred to above in 


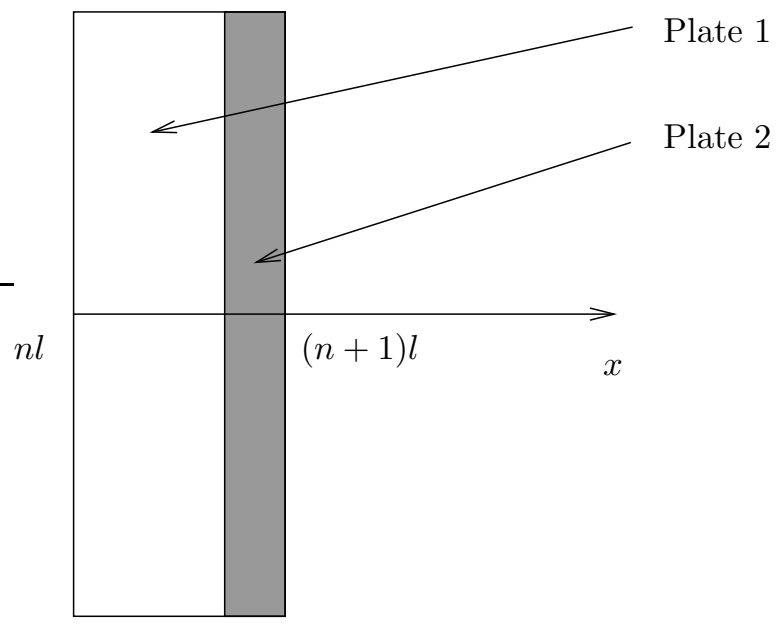

Figure 2: Figure depicting an example of the $n$th periodic cell in the one dimensional medium.

the heat equation even though the class of problems considered certainly require the use of the fully coupled theory.

It appears that no homogenized coupling condition analogous to (1.5) (and hence no effective coupling parameter $\delta_{*}$ ) has yet been defined in the literature for effective media and this issue will be addressed in this article. Boundary conditions imposed on the surface of the half-space at $x=0$ will lead to strain and temperature rates which do not satisfy such an effective coupling condition and thus will require the fully coupled theory.

The effective properties of layered elastic media are well understood, see for example [17], [14] and references therein. Thermoelastic composites were apparently first studied by Kerner [10] and Budiansky [7] who were concerned with matrix/inclusion composites. Laws [11] discussed the thermostatic case and Francfort [9] provided a rigorous analysis of the homogenization of thermoelastic media which although fairly complete does not provide any results regarding the effective coupling parameter. Most other work ignores coupling completely. Furthermore, it does not appear that any transient, coupled thermoelastic composite problems have been studied until now.

The purpose of the present article is therefore firstly to carry out the homogenization of the fully coupled thermoelastic problem systematically via the method of asymptotic homogenization in order to obtain the homogenized system $(\epsilon \rightarrow 0)$ and identify effective coefficients. Secondly we shall solve a class of transient boundary value problems for the homogenized half space which requires fully coupled thermoelasticity. We compare the results with the classical homogeneous problem solved by Boley and Tolins [4].

The homogenization step leads to some interesting findings regarding the effective coupling parameter of the homogenized medium. In particular it is shown that for a two-phase composite the coupling parameter is not bounded below by the coupling parameters corresponding to the two homogeneous phases which make up the composite.

For the transient problems considered, the boundary conditions imposed on 
$x=0$ lead to slowly varying field variables except at a single point where in some cases they are discontinuous. Since the microscale $l$ of the composite material will always remain finite (but small) in practice, the validity of the homogenization theory could therefore be called into question. Indeed homogenization theory is not strictly valid in such a case precisely for this reason. We reconcile this difficulty as follows. As discussed, homogenization is valid for dynamic problems provided the separation of scales inequalities hold:

$$
l \ll \eta, \quad l \ll L .
$$

A discontinuous solution would not satisfy the first of these inequalities since $\eta=0$. Consider then a smoothed out discontinuity over a finite lengthscale $\eta \gg l$. We are interested in the homogenized limit when $\epsilon=l / \eta \rightarrow 0$. Therefore the discontinuous solution can be thought of as the limiting solution when $\eta \rightarrow 0$ in the smoothed out solution and $l \rightarrow 0$ such that we retain $l \ll \eta$. This is easily achieved by choosing say $l=O\left(\Delta^{2}\right)$ and $\eta=O(\Delta)$ where $\Delta \rightarrow 0$ and thus $\epsilon=l / \eta=O(\Delta)$. Such a smoothed out solution will still require coupling effects to be taken into account since the variation of the propagating field in the vicinity of the propagating front is rapid with respect to the lengthscale of the body. The limiting process ensures however that it is slowly varying with respect to the microscale.

The "step" boundary value problems considered in section 5 (leading to the propagation of a thermoelastic discontinuity) are considered for simplicity and the homogenized solutions should be considered as the limit of the limiting process above as $\Delta \rightarrow 0$ which is the strict homogenization regime. They provide us with qualitative information about the behaviour of solutions for finite $l$ and slightly smoother boundary conditions where coupling effects are still important. The homogenization scheme developed shortly is therefore valid for all classes of such materials, provided that the propagating fields have variations in lengthscale satisfying (1.7).

When the first of the inequalities (1.7) is not satisfied, more complicated physics ensues in the composite. This cannot be described by a set of homogenized equations. For periodic media, stop and pass bands arise and it can be particularly useful to exploit this phenomenon [16]. For slightly non-periodic media, the important issue is that of Anderson localization [6].

The article will proceed as follows: In section 2 we shall apply the method of asymptotic homogenization in order to obtain the homogenized governing equations, valid in the limit as $\epsilon \rightarrow 0$ and we shall define the effective properties. We consider the nondimensionalization of this system in section 3 and thus define the effective coupling parameter. Effective properties are studied in more detail in section 4 . The necessary boundary conditions are then introduced. In order to compare results with those obtained for the homogeneous half-space considered by Boley and Tolins [4] we consider the mathematically convenient problem of specifying strain and temperature boundary conditions.

We outline the solution method for the homogenized system in section 5 . Given initial conditions of zero temperature, displacement and rate of displacement in $x>0$ we solve two problems in the transformed domain. The full solution in the physical domain is determined in section 6 , where we analyse the magnitude of the resulting discontinuities in the field variables and their derivatives. 
In section 7 we analyse the more physically meaningful situation of imposing stress and temperature boundary conditions (Danilovskaya's problem) and show that by linearity these may be obtained by adding solutions determined in section 6 . We close in section 8 , summarising our findings and indicating areas for further study.

\section{Homogenization}

We shall introduce the relative temperature $T_{\epsilon}$ in the governing equations (1.1) and (1.4), defined by

$$
T_{\epsilon}(x, t)=\Theta_{\epsilon}(x, t)-\Theta_{0}
$$

and these fluctuations must be small compared with $\Theta_{0}$ since we are in the linear regime. The initial conditions of the problem are

$$
\begin{gathered}
u_{\epsilon}(x>0, t=0)=0, \quad \frac{\partial u_{\epsilon}}{\partial t}(x>0, t=0)=0, \\
T_{\epsilon}(x>0, t=0)=0 .
\end{gathered}
$$

We will consider boundary conditions once we have homogenized the governing equations and nondimensionalized the resulting system. We apply the method of asymptotic homogenization [3], [12], [13] which requires the introduction of the independent variables

$$
x=z, \quad \xi=\frac{x}{\epsilon},
$$

so that from (1.1) and (1.4) we see that the material properties are 1-periodic functions of $\xi$. Note that we are not restricting attention here to the laminated composite depicted in figure 1 . The derivative with respect to $x$ becomes

$$
\frac{d}{d x}=\frac{\partial}{\partial z}+\frac{1}{\epsilon} \frac{\partial}{\partial \xi}
$$

Thus, on expanding the displacement and temperature in asymptotic expansions of the form

$$
\begin{aligned}
& u_{\epsilon}(x, t)=u_{0}(z, \xi, t)+\epsilon u_{1}(z, \xi, t)+\epsilon^{2} u_{2}(z, \xi, t)+\ldots \\
& T_{\epsilon}(x, t)=T_{0}(z, \xi, t)+\epsilon T_{1}(z, \xi, t)+\epsilon^{2} T_{2}(z, \xi, t)+\ldots
\end{aligned}
$$

the governing equations (1.1) and (1.4) become

$$
\begin{aligned}
\left(\frac{\partial}{\partial z}+\frac{1}{\epsilon} \frac{\partial}{\partial \xi}\right)(k(\xi) & \left.\left(\frac{\partial}{\partial z}+\frac{1}{\epsilon} \frac{\partial}{\partial \xi}\right)\left(T_{0}+\epsilon T_{1}+\epsilon^{2} T_{2}+\ldots\right)\right) \\
= & \rho(\xi) c_{v}(\xi) \frac{\partial}{\partial t}\left(T_{0}+\epsilon T_{1}+\epsilon^{2} T_{2}+\ldots\right) \\
+(3 \lambda(\xi) & +2 \mu(\xi)) \alpha(\xi) \Theta_{0}\left(\frac{\partial}{\partial z}+\frac{1}{\epsilon} \frac{\partial}{\partial \xi}\right) \frac{\partial}{\partial t}\left(u_{0}+\epsilon u_{1}+\ldots\right)
\end{aligned}
$$




$$
\begin{array}{r}
\left(\frac{\partial}{\partial z}+\frac{1}{\epsilon} \frac{\partial}{\partial \xi}\right)\left((\lambda(\xi)+2 \mu(\xi))\left(\frac{\partial}{\partial z}+\frac{1}{\epsilon} \frac{\partial}{\partial \xi}\right)\left(u_{0}+\epsilon u_{1}+\epsilon^{2} u_{2}+\ldots\right)\right) \\
-\left(\frac{\partial}{\partial z}+\frac{1}{\epsilon} \frac{\partial}{\partial \xi}\right)\left((3 \lambda(\xi)+2 \mu(\xi)) \alpha(\xi)\left(T_{0}+\epsilon T_{1}+\epsilon^{2} T_{2}+\ldots\right)\right) \\
=\rho(\xi) \frac{\partial^{2}}{\partial t^{2}}\left(u_{0}+\epsilon u_{1}+\ldots\right)
\end{array}
$$

and the initial conditions are

$$
\begin{aligned}
\left(u_{0}+\epsilon u_{1}+\ldots\right)(x>0, t & =0)=0, \\
\frac{\partial}{\partial t}\left(u_{0}+\epsilon u_{1}+\ldots\right)(x>0, t & =0)=0, \\
\left(T_{0}+\epsilon T_{1}+\ldots\right)(x>0, t & =0)=0 .
\end{aligned}
$$

The periodicity of the microstructure implies that

$$
T_{j}(z, \xi, t)=T_{j}(z, \xi+1, t), \quad u_{j}(z, \xi, t)=u_{j}(z, \xi+1, t),
$$

for $j=0,1,2, \ldots$. We define the operation of averaging a function $f(z, \xi, t)$ over the periodic cell as:

$$
\langle f\rangle=\int_{0}^{1} f(z, \xi, t) d \xi .
$$

On equating orders at $O\left(\epsilon^{-2}\right)$ we deduce that $O(1)$ functions are independent of the microscale $\xi$ :

$$
T_{0}=T_{0}(z, t), \quad u_{0}=u_{0}(z, t) .
$$

At $O\left(\epsilon^{-1}\right)$ we have

$$
\begin{gathered}
\frac{\partial}{\partial \xi}\left(k(\xi)\left(\frac{\partial T_{0}}{\partial z}+\frac{\partial T_{1}}{\partial \xi}\right)\right)=0 \\
\frac{\partial}{\partial \xi}\left[(\lambda(\xi)+2 \mu(\xi))\left(\frac{\partial u_{0}}{\partial z}+\frac{\partial u_{1}}{\partial \xi}\right)-(3 \lambda(\xi)+2 \mu(\xi)) \alpha(\xi) T_{0}\right]=0 .
\end{gathered}
$$

From equation (2.16) and on using the first of (2.13) with $j=1$, we obtain

$$
T_{1}(z, \xi, t)=N_{1}(\xi) \frac{\partial T_{0}}{\partial z}
$$

where

$$
N_{1}(\xi)=\int_{c_{1}}^{\xi}\left(\frac{k_{*}}{k(s)}-1\right) d s, \quad k_{*}=\left(\int_{0}^{1} \frac{1}{k(s)} d s\right)^{-1},
$$

and where $c_{1}$ is a constant to be determined from the forthcoming boundary conditions. From (2.17) we obtain

$$
u_{1}(z, \xi, t)=M_{1}(\xi) \frac{\partial u_{0}}{\partial z}-P_{1}(\xi) T_{0}
$$


where

$$
\begin{aligned}
M_{1}(\xi) & =\int_{c_{2}}^{\xi} \frac{1}{R_{2}}\left(\frac{1}{\lambda(s)+2 \mu(s)}-R_{2}\right) d s, \\
P_{1}(\xi) & =\int_{c_{3}}^{\xi} \frac{1}{R_{2}(\lambda(s)+2 \mu(s))}\left(R_{2} \alpha(s)(3 \lambda(s)+2 \mu(s))-R_{1}\right) d s, \\
R_{1} & =\left\langle\frac{(3 \lambda(\xi)+2 \mu(\xi)) \alpha(\xi)}{\lambda(\xi)+2 \mu(\xi)}\right\rangle, \\
R_{2} & =\left\langle\frac{1}{\lambda(\xi)+2 \mu(\xi)}\right\rangle,
\end{aligned}
$$

and $c_{2}$ and $c_{3}$ are constants to be determined from boundary conditions.

In order to find the effective coefficients of the homogenized system and the leading order (homogenized) solutions, it is not necessary to determine the constants $c_{1}, c_{2}$ and $c_{3}$. These constants are calculated by imposing higher order boundary conditions on $x=0$ and therefore appear in the first order correction to the homogenized solution. This first order term therefore provides an estimate of the error we are making when assuming a homogenized composite. Very little work of this nature regarding the connection between this error and boundary conditions exists but see [15] for further discussion. Work is underway to see what implications this has for the type of half-space problems considered here both in an elastic and thermoelastic context.

On averaging the $O(1)$ terms of (2.8) over a periodic cell we find that

$$
\begin{aligned}
k_{*} \frac{\partial^{2} T_{0}}{\partial z^{2}}=\left\langle\rho(\xi) c_{v}(\xi)\right\rangle(1+\langle\gamma(\xi) \beta(\xi)\rangle) & \frac{\partial T_{0}}{\partial t}+ \\
& \frac{\Theta_{0}}{R_{2}}\left\langle\frac{3 \lambda(\xi)+2 \mu(\xi)}{\lambda(\xi)+2 \mu(\xi)} \alpha(\xi)\right\rangle \frac{\partial^{2} u_{0}}{\partial t \partial x}
\end{aligned}
$$

where

$$
\begin{aligned}
& \gamma(\xi)=\frac{\Theta_{0} \alpha^{2}(\xi)(3 \lambda(\xi)+2 \mu(\xi))^{2}}{\left\langle\rho(\xi) c_{v}(\xi)\right\rangle(\lambda(\xi)+2 \mu(\xi))}, \\
& \beta(\xi)=1-\frac{R_{1}}{R_{2} \alpha(\xi)(3 \lambda(\xi)+2 \mu(\xi))} .
\end{aligned}
$$

The quantity $\beta(\xi)$ is a measure of material differences and is zero for a homogeneous material. Thus we may write $(2.25)$ as

$$
\frac{\partial^{2} T_{0}}{\partial z^{2}}=\frac{1}{D_{*}} \frac{\partial T_{0}}{\partial t}+\frac{R_{0} \Theta_{0}}{k_{*}} \frac{\partial^{2} u_{0}}{\partial t \partial z}
$$

where

$$
\begin{aligned}
D_{*} & =\frac{k_{*}}{S_{*}}, \\
S_{*} & =\left\langle\rho(\xi) c_{v}(\xi)\right\rangle(1+\langle\gamma(\xi) \beta(\xi)\rangle), \\
R_{0} & =\frac{1}{R_{2}}\left\langle\frac{3 \lambda(\xi)+2 \mu(\xi)}{\lambda(\xi)+2 \mu(\xi)} \alpha(\xi)\right\rangle .
\end{aligned}
$$


It is interesting to note that $S_{*}$, which may be interpreted as the effective heat capacity (per unit volume) of the inhomogeneous medium, is not simply the average of $\rho(\xi) c_{v}(\xi)$. We may interpret $D_{*}$ and $k_{*}$ as the effective thermal diffusivity and conductivity of the medium respectively. The behaviour of these effective properties will be considered in section 4 .

Similarly, averaging the $O(1)$ terms of equation (2.9) over a periodic cell and simplifying, we obtain the homogenized equation of motion

$$
\frac{\partial^{2} u_{0}}{\partial z^{2}}=R_{1} \frac{\partial T_{0}}{\partial z}+R_{2} \rho_{*} \frac{\partial^{2} u_{0}}{\partial t^{2}}
$$

where

$$
\rho_{*}=\langle\rho(\xi)\rangle
$$

is the usual form for the effective static mass density [3]. Finally, the initial conditions at $O(1)$ are

$$
\begin{gathered}
u_{0}(z>0, t=0)=0, \\
\frac{\partial u_{0}}{\partial t}(z>0, t=0)=0, \\
T_{0}(z>0, t=0)=0 .
\end{gathered}
$$

We shall now nondimensionalize the governing homogenized equations and introduce the boundary conditions on the surface of the half space.

\section{Scaling, Boundary Conditions and the Effect- ive Coupling Parameter}

We are interested in solving the homogenized system comprising equations (2.28) and (2.32) subject to the initial conditions (2.34)-(2.36) and boundary conditions which will be specified shortly.

In order to nondimensionalize we choose the following scalings:

$$
\begin{aligned}
\hat{z} & =\frac{v_{*}}{D_{*}} z, & \hat{u}_{\epsilon} & =\frac{v_{*}}{D_{*} R_{1} \Theta_{0}} u_{\epsilon}, \\
\hat{t} & =\frac{v_{*}^{2}}{D_{*}} t, & \hat{T}_{\epsilon} & =\frac{T_{\epsilon}}{\Theta_{0}}, \\
\hat{e}_{\epsilon} & =\frac{e_{\epsilon}}{R_{1} \Theta_{0}}, & \hat{\sigma}_{\epsilon} & =\frac{R_{2}}{R_{1} \Theta_{0}} \sigma_{\epsilon},
\end{aligned}
$$

where we have defined

$$
v_{*}^{2}=\frac{1}{R_{2} \rho_{*}}
$$

which as we shall see is the square of the velocity of propagating discontinuities in the homogenized medium. Introducing these scalings into (2.28) and (2.32), and dropping hats on the nondimensionalized variables, the system becomes

$$
\begin{aligned}
& \frac{\partial^{2} T_{0}}{\partial z^{2}}=\frac{\partial T_{0}}{\partial t}+\delta_{*} \frac{\partial^{2} u_{0}}{\partial t \partial z} \\
& \frac{\partial^{2} u_{0}}{\partial z^{2}}=\frac{\partial T_{0}}{\partial z}+\frac{\partial^{2} u_{0}}{\partial t^{2}}
\end{aligned}
$$


where we have defined the effective coupling parameter

$$
\begin{aligned}
\delta_{*} & =\frac{1}{k_{*}} R_{1} D_{*} R_{0} \Theta_{0} \\
& =\frac{\Theta_{0}\left\langle(\lambda(\xi)+2 \mu(\xi))^{-1}\right\rangle^{-1}}{\left\langle\rho(\xi) c_{v}(\xi)\right\rangle(1+\langle\gamma(\xi) \beta(\xi)\rangle)}\left\langle\frac{(3 \lambda(\xi)+2 \mu(\xi)) \alpha(\xi)}{(\lambda(\xi)+2 \mu(\xi))}\right\rangle^{2} .
\end{aligned}
$$

This parameter reduces to the usual form for homogeneous media (cf. Boley and Tolins' [4], equation (28)) and exhibits some very interesting behaviour for inhomogeneous media as is discussed in section 4 . We can now state the analogous (nondimensionalized) condition for inhomogeneous media to (1.5). The dilatational coupling in the effective, modified heat equation (3.5) may be neglected if

$$
\frac{\partial^{2} u_{0}}{\partial z \partial t} / \frac{\partial T_{0}}{\partial t} \ll \frac{1}{\delta_{*}} .
$$

As in the case of homogeneous media, the effective coupling constant will also usually satisfy $\delta_{*} \ll 1$, but as indicated earlier, the coupling can only be neglected if

$$
\frac{\partial^{2} u_{0}}{\partial z \partial t} \sim \frac{\partial T_{0}}{\partial t}
$$

in the homogenized medium. Notice furthermore that this condition is different from that of homogeneous media since in a homogeneous medium, $\partial u_{0} / \partial z$ is the strain whereas in our homogenized medium this is not the case as we discuss in section 3.1 shortly.

On nondimensionalization, the initial conditions (2.34)-(2.36) do not change form since they are homogeneous. In order to compare results with those obtained for the homogeneous medium by Boley and Tolins [4] we shall consider boundary conditions which impose strain and temperature on $z=0$. In section 7 we shall consider what these mean in terms of the more physically meaningful traction and temperature boundary conditions and thus we note that the leading order nondimensional stress is given by

$$
\sigma_{0}=\frac{\partial u_{0}}{\partial z}-T_{0} .
$$

\subsection{Strain and Temperature Flux}

It is important to note that although the displacement and temperature are homogenized and thus depend only on $z$, the strain and temperature flux will depend on the microscale variable $\xi$. The leading order nondimensional strain is given by

$$
\begin{aligned}
e_{0}(z, \xi, t)= & \frac{\partial u_{0}}{\partial z}(z, t)+\frac{\partial u_{1}}{\partial \xi}(z, \xi, t) \\
= & \frac{1}{R_{2}(\lambda(\xi)+2 \mu(\xi))} \frac{\partial u_{0}}{\partial z}(z, t) \\
& +\left(\frac{R_{2}(3 \lambda(\xi)+2 \mu(\xi)) \alpha(\xi)-R_{1}}{R_{1} R_{2}(\lambda(\xi)+2 \mu(\xi))}\right) T_{0}(z, t),
\end{aligned}
$$


having used (2.20). Furthermore on using (2.18), the leading order temperature flux is given by

$$
Q_{0}(z, \xi, t)=\frac{\partial T_{0}}{\partial z}(z, t)+\frac{\partial T_{1}}{\partial \xi}(z, \xi, t)=\frac{k_{*}}{k(\xi)} \frac{\partial T_{0}}{\partial z}(z, t) .
$$

\subsection{Boundary Conditions}

We pose the following (nondimensionalized) strain and temperature boundary conditions:

$$
\begin{array}{r}
e_{\epsilon}(z=0, \xi=0, t)=\frac{\partial u_{\epsilon}}{\partial x}(z=0, \xi=0, t)=f(t), \\
T_{\epsilon}(z=0, \xi=0, t)=g(t)
\end{array}
$$

where $f(t)$ and $g(t)$ are $O(1)$ functions. The strain boundary condition at $O\left(\epsilon^{-1}\right)$ is automatically satisfied since $u_{0}=u_{0}(z, t)$. At $O(1)$ the boundary conditions become

$$
\begin{array}{r}
e_{0}(z=0, \xi=0, t)=\frac{\partial u_{0}}{\partial z}(z=0, t)+\frac{\partial u_{1}}{\partial \xi}(z=0, \xi=0, t)=f(t) \\
T_{0}(z=0, t)=g(t)
\end{array}
$$

and on using (3.13) these conditions are

$$
\begin{aligned}
\frac{\partial u_{0}}{\partial z}(z & =0, t)=c f(t)-h g(t), \\
T_{0}(z=0, t) & =g(t),
\end{aligned}
$$

where

$$
\begin{aligned}
c & =R_{2}(\lambda(0)+2 \mu(0)), \\
h & =\left(\frac{R_{2}}{R_{1}}(3 \lambda(0)+2 \mu(0)) \alpha(0)-1\right),
\end{aligned}
$$

and $h$ is the homogeneity parameter. Note that for a homogeneous material having material moduli $\lambda(0), \mu(0)$, etc. we find that

$$
c \rightarrow 1, \quad h \rightarrow 0,
$$

as should be the case. We will shortly provide a summary of the $O(1)$ problem which we shall solve, but first let us consider the effective thermoelastic properties obtained above.

\section{Effective Thermoelastic Properties}

Many of the effective properies such as the effective density, conductivity, etc. are already relatively well understood and thus here we focus on effective coefficients possessing interesting and novel properties. Consider a composite consisting of laminated plates (as depicted in figure 1) with two materials (Lockalloy and Epoxy) in the periodic cell, having the properties given in table 1, taken from Baczynski [2]. 


\begin{tabular}{lcc}
\hline & Lockalloy & Epoxy \\
\hline Mass density $\left(\mathrm{kg} / \mathrm{m}^{3}\right)$ & $\rho_{0}=2090$ & $\rho_{1}=1149$ \\
Specific heat $(\mathrm{J} / \mathrm{kgK})$ & $c_{v 0}=1653$ & $c_{v 1}=1883$ \\
Thermal conductivity $(\mathrm{W} / \mathrm{mK})$ & $k_{0}=212.74$ & $k_{1}=0.35$ \\
Elastic Moduli $\left(\mathrm{kG} / \mathrm{m}^{2}\right)$ & $\lambda_{0}=0.32 \times 10^{9}$ & $\lambda_{1}=3.12 \times 10^{9}$ \\
& $\mu_{0}=74 \times 10^{9}$ & $\mu_{1}=1.09 \times 10^{9}$ \\
Thermal expansion coeff $(1 / \mathrm{K})$ & $\alpha_{0}=23 \times 10^{-6}$ & $\alpha_{1}=57 \times 10^{-6}$ \\
Coupling parameter & 0.00687223 & 0.0113197 \\
\hline
\end{tabular}

Table 1: Material properties of the phases comprising the composite at the reference temperature $\Theta_{0}=300^{\circ} \mathrm{K}$. Note the coupling parameter associated with each phase.

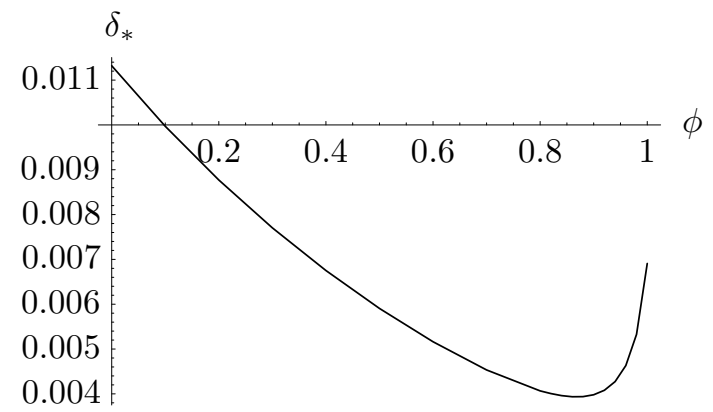

Figure 3: Figure showing the dependence of the effective coupling constant on volume fraction of the Lockalloy phase. In particular note the non-monotonicity and the fact that the composite has coupling coefficients below that of either the Epoxy or Lockalloy phases for a wide range of volume fractions.

\subsection{Effective Coupling Parameter}

The crucial parameter regarding the thermoelastic coupling is the effective coupling parameter $\delta_{*}$ defined in (3.8). We plot this against volume fraction of the Lockalloy phase in figure 3 . Note in particular the non-monotonicity and the fact that in certain volume fraction ranges the coupling constant decreases below that which would occur in a homogeneous material of either the Lockalloy or Epoxy phases. This behaviour is certainly not what one may expect and indeed it could even be exploited in order to minimize the amount of thermal coupling in the material. It is seen here that $\delta_{*}$ is at its minimum $\left(\delta_{*} \approx 0.0039\right)$ at $\phi \approx 0.87$.

In all cases of two phase materials considered by the author it appears that $\max \{\delta(\xi)\}$ provides an upper bound on $\delta_{*}$ but as is exhibited by the case above $\min \{\delta(\xi)\}$ does not provide a lower bound. We have not yet been able to prove this rigorously and work is underway to establish such a result. Note that what leads to this result is the fact that the harmonic average of $\lambda(\xi)+2 \mu(\xi)$ arises in the expression for $\delta_{*}$ rather than a simple volume average. In order to establish what effect this has, let us pose the following expression for $\delta_{*}=\delta_{*}^{v}$, instead of 


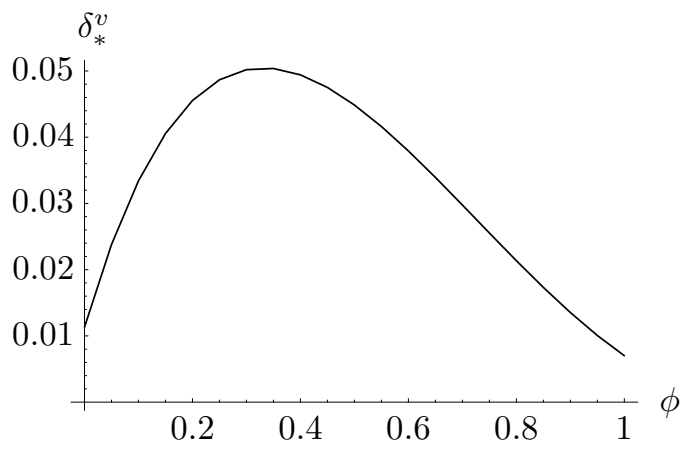

Figure 4: Plot of the effective coupling parameter $\delta_{*}^{v}$ posed in (4.1) involving volume averages. Note that this possesses completely different behaviour from the true effective coupling constant $\delta_{*}$.

$$
\delta_{*}^{v}=\frac{\Theta_{0}\langle\lambda(\xi)+2 \mu(\xi)\rangle}{\left\langle\rho(\xi) c_{v}(\xi)\right\rangle(1+\langle\gamma(\xi) \beta(\xi)\rangle)}\left\langle\frac{(3 \lambda(\xi)+2 \mu(\xi)) \alpha(\xi)}{(\lambda(\xi)+2 \mu(\xi))}\right\rangle^{2}
$$

which involves the volume average of $\lambda(\xi)+2 \mu(\xi)$. Plotting this in figure 4 for the Lockalloy/Epoxy composite, we see that it exhibits completely different behaviour to the correctly defined $\delta_{*}$ given by (3.8) and plotted in figure 3 . In particular in this case the amount of coupling is increased for a wide range of volume fractions.

We often encounter the harmonic average in effective media theories [3] and this provides yet another example of how important it is to carry out the homogenization properly, as above, rather than with some ad hoc volume averaging argument.Work on more general composites and their effective properties, including the effective coupling parameter, is currently underway.

\subsection{Effective Heat Capacity}

The effective heat capacity $S_{*}$ (defined in (2.30)) for the Lockalloy/Epoxy composite is shown in figure 5 . It shows a clear linear dependence on the volume fraction. In figure 6 we illustrate the difference between the true effective heat capacity and the naïve approximation given by $\left\langle\rho c_{v}\right\rangle$. Note that this is at a maximum where the coupling constant is at a minimum. The magnitude of the difference between the true effective heat capacity and $\left\langle\rho c_{v}\right\rangle$ is highly dependent on the coefficients of thermal expansion of the two phases.

\subsection{Effective Diffusivity}

Next, let us consider the effective thermal diffusivity $D_{*}$, defined in (2.29). In figure 7 this quantity is plotted against volume fraction. Note that even for high volume fractions of the Lockalloy phase, a low thermal diffusivity is achieved due to the much lower thermal conductivity of the Epoxy phase. 


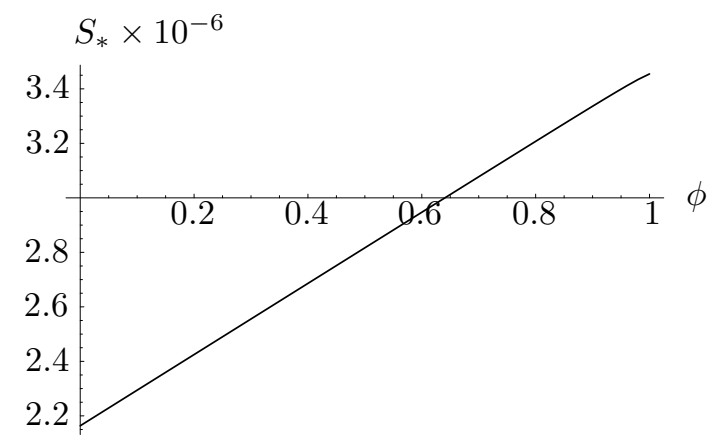

Figure 5: Figure showing the linear dependence of the effective heat capacity on the volume fraction $\phi$ of the Lockalloy phase.

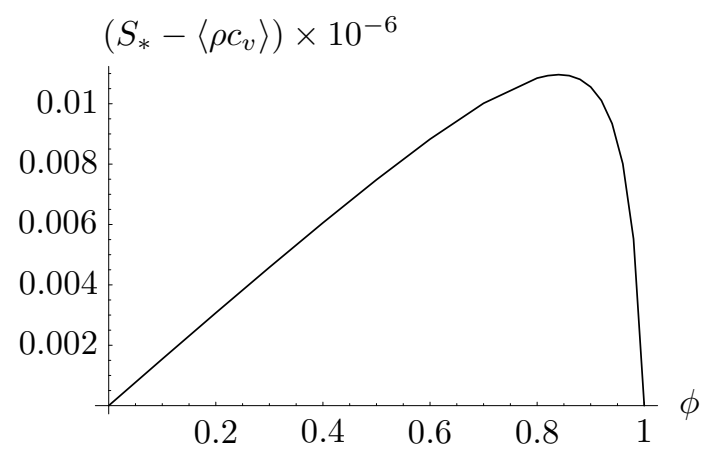

Figure 6: Figure showing the difference between the true effective heat capacity $S_{*}$ and a naive approximation $\left\langle\rho c_{v}\right\rangle$. Note that this difference is at a maximum at the same volume fraction where the coupling constant takes a minimum. We note from figure 5 that the effective heat capacity $S_{*}$ remains monotonic.

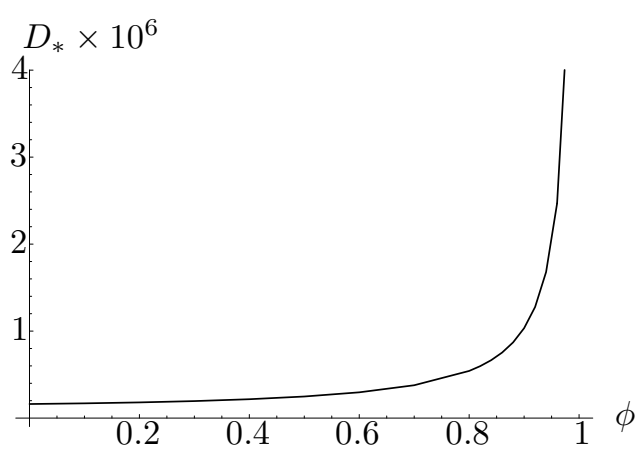

Figure 7: Figure showing the dependence of the effective thermal diffusivity on volume fraction of the Lockalloy phase. Note that due to the much lower thermal conductivity of the Epoxy phase, it takes a very high volume fraction of the Lockalloy phase before the effective thermal diffusivity is increased much above that of the Epoxy phase. 


\subsection{Effective Propagation Speed}

We shall show in the sections to follow that discontinuities propagate through the homogenized medium with the effective propagation speed $v_{*}$ defined in (3.4), which for the composite considered here is plotted in figure 8.

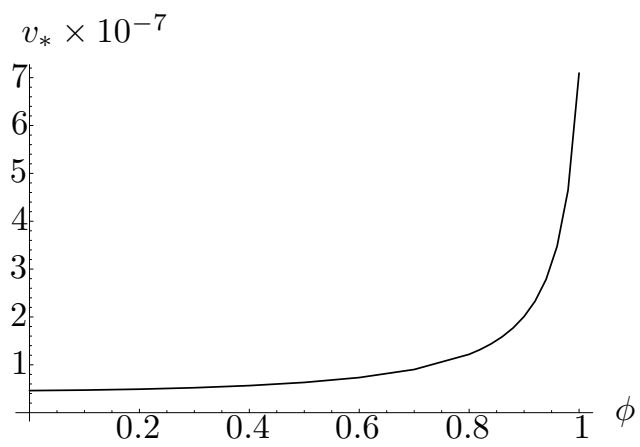

Figure 8: Figure showing the dependence of the effective speed of propagation of discontinuities in the solutions (see section 6) for the homogenized half space.

\section{Solution Method}

\section{Nondimensionalized Summary}

The homogenized system is:

$$
\begin{gathered}
\frac{\partial^{2} u_{0}}{\partial z^{2}}=\frac{\partial T_{0}}{\partial z}+\frac{\partial^{2} u_{0}}{\partial t^{2}}, \\
\frac{\partial^{2} T_{0}}{\partial z^{2}}=\frac{\partial T_{0}}{\partial t}+\delta_{*} \frac{\partial^{2} u_{0}}{\partial t \partial z}, \\
\frac{\partial u_{0}}{\partial z}(z=0, t)=c f(t)-h g(t), \quad T_{0}(z=0, t)=g(t), \\
u_{0}(z>0, t=0)=0, \quad \frac{\partial u_{0}}{\partial t}(z>0, t=0)=0, \\
T_{0}(z>0, t=0)=0,
\end{gathered}
$$

which may now be solved in a similar manner to the corresponding system of Boley and Tolins [4] by using the following cosine and sine transform pairs:

$$
\begin{array}{rlrl}
U(p, t) & =\int_{0}^{\infty} u_{0}(z, t) \cos p z d z, & u_{0}(z, t)=\frac{2}{\pi} \int_{0}^{\infty} U(p, t) \cos p z d z, \\
V(p, t)=\int_{0}^{\infty} T_{0}(z, t) \sin p z d z, & T_{0}(z, t)=\frac{2}{\pi} \int_{0}^{\infty} V(p, t) \sin p z d z,
\end{array}
$$

which decouples the system into two third order ordinary differential equations. We refer the reader to [4] for details. Here we discuss the resulting homogenized fields for the following two problems.

\section{Problem 1}


Boundary conditions are specified for $t \geq 0$ by

$$
f(t)=\epsilon_{0}, \quad g(t)=0,
$$

where $\epsilon_{0} \in \mathbb{R}$ is a constant and thus for $t \geq 0$ we have

$$
\frac{\partial u_{0}}{\partial z}(z=0, t)=c \epsilon_{0}, \quad T_{0}(z=0, t)=0 .
$$

\section{Problem 2}

$$
f(t)=0, \quad g(t)=\theta_{0},
$$

where $\theta_{0} \in \mathbb{R}$ is a constant and thus for $t \geq 0$ we have

$$
\frac{\partial u_{0}}{\partial z}(z=0, t)=-h \theta_{0}, \quad T_{0}(z=0, t)=\theta_{0} .
$$

Note that for a homogeneous medium $(h=0)$, these two problems together with the condition that $\theta_{0}=\delta_{*} \epsilon_{0}$ satisfy Boley and Tolins' reciprocal relation. In the inhomogeneous context considered here however, the coupling in the boundary condition of Problem 2 means that this reciprocal relation cannot hold.

As we shall see the boundary conditions above lead to discontinuous homogenized displacement gradient fields. As discussed in the introduction, such homogenized fields are only valid in the homogenization limit when $\Delta \rightarrow 0$.

Now, provided that

$$
\delta_{*} \leq-10+\sqrt{108} \approx 0.4
$$

(the common case in most materials) there are one real negative and two complex conjugate roots [4] of the characteristic equation corresponding to the homogeneous (zero right hand side) versions of the resulting ordinary differential equations referred to above. Following Boley and Tolins, we denote these roots as

$$
m_{1}=-\alpha, \quad m_{2}=-\beta+i \gamma, \quad m_{3}=-\beta-i \gamma,
$$

where $\alpha, \beta$ and $\gamma$ are positive and real. Thus, on finding the correct particular solution to the ordinary differential equations we find the following solutions in the transformed domain.

\section{Problem 1}

$$
\begin{aligned}
& \begin{array}{r}
\frac{U(p, t)}{c \epsilon_{0}}=\frac{1}{\left((\alpha-\beta)^{2}+\gamma^{2}\right)}\left(\frac{2 \beta}{\alpha} \exp (-\alpha t)\right. \\
+\frac{\alpha\left(\alpha^{2}+\gamma^{2}-3 \beta^{2}\right)}{p^{4}} \exp (-\beta t) \cos \gamma t \\
\left.\quad+\frac{\alpha \beta\left(\alpha^{2}-\beta^{2}+3 \gamma^{2}\right)}{\gamma p^{4}} \exp (-\beta t) \sin \gamma t\right)-\frac{1}{p^{2}} \\
\frac{V(p, t)}{c \epsilon_{0}}=\frac{-\delta_{*} p}{\left((\alpha-\beta)^{2}+\gamma^{2}\right)}(\exp (-\alpha t)-\exp (-\beta t) \cos \gamma t \\
\left.\quad+\frac{(\alpha-\beta)}{\gamma} \exp (-\beta t) \sin \gamma t\right) .
\end{array}
\end{aligned}
$$


Note that this solution is identical to the corresponding problem in [4] except that here the solutions are functions of the effective coupling parameter $\delta_{*}$.

\section{Problem 2}

$$
\begin{gathered}
\frac{U(p, t)}{\theta_{0}}=\frac{(1+h)}{\left((\alpha-\beta)^{2}+\gamma^{2}\right)}\left(\left(1-\frac{h p^{2}}{\alpha}\right) \exp (-\alpha t)\right. \\
\quad-\left(1+\frac{h \alpha(\alpha-2 \beta)}{p^{2}}\right) \exp (-\beta t) \cos \gamma t \\
\left.+\left(\frac{\alpha-\beta}{\gamma}-\frac{h \alpha}{\gamma}\left(1+\delta_{*}-\beta\right)\right) \exp (-\beta t) \sin \gamma t\right)+\frac{h}{p^{2}}, \\
\frac{V(p, t)}{\theta_{0}}=\frac{1}{p\left((\alpha-\beta)^{2}+\gamma^{2}\right)}\left(-\left(\alpha^{2}+(1-h) p^{2}\right) \exp (-\alpha t)\right. \\
\quad+\left(4 \alpha \beta-\left(\delta_{*}+h\right) p^{2}\right) \exp (-\beta t) \cos \gamma t \\
\left.+\frac{p^{2}}{\gamma}\left(2 \beta(1-\alpha)+\delta_{*}(\alpha+\beta)+h(\alpha-\beta)\right) \exp (-\beta t) \sin \gamma t\right)
\end{gathered}
$$

which is considerably different to its homogeneous counterpart in [4] and recovers the form for the homogeneous material as $h \rightarrow 0$.

\section{Solution in the Physical Domain and Analysis of the Propagation of Discontinuities}

Having obtained the solutions in the transformed domains we now obtain the full solutions in an integral form convenient for numerical evaluation and one that exhibits the form of discontinuities in the solutions, by using the cosine and sine transform inversion integrals. Convenient forms are found by subtracting the behaviour of the solutions as $p \rightarrow \infty$ from the integrands. Details of this behaviour and definitions of the functions $R_{j}(t)$ and $S_{j}(t), j=1,2,3$ are given in the appendix.

\section{Problem 1}

We can write the inversion integrals in the form

$$
\begin{array}{r}
\frac{1}{c \epsilon_{0}} \frac{\partial u_{0}}{\partial z}(z, t)= \\
-\frac{2}{\pi} \int_{0}^{\infty} p\left(U(p, t)-R_{1}(t) \frac{\cos p t}{p^{2}}-S_{1}(t) \frac{\sin p t}{p^{3}}+\frac{1}{p^{2}}\right) \sin p z d p \\
+F_{1}(z, t)+G_{1}(z, t)+1, \\
\frac{1}{c \epsilon_{0}} T_{0}(z, t)=\frac{2}{\pi} \int_{0}^{\infty}\left(T(p, t)-R_{2}(t) \frac{\sin p t}{p^{2}}\right) \sin p z d p \\
+F_{2}(z, t),
\end{array}
$$


where unity has appeared in (6.1) since we have removed the particular integral $-1 / p^{2}$ from the displacement and have thus added on the term

$$
\frac{2}{\pi} \int_{0}^{\infty} \frac{\sin p z}{p} d p=1, \quad \text { for } z>0 .
$$

Also

$$
\begin{aligned}
F_{1}(z, t) & =-R_{1}(t) \mathcal{I}_{c}(z, t) \\
G_{1}(z, t) & =-S_{1}(t) \mathcal{I}_{s}(z, t) \\
F_{2}(z, t) & =R_{2}(t) \mathcal{I}_{s}(z, t)
\end{aligned}
$$

where

$$
\begin{aligned}
& \mathcal{I}_{c}(z, t)=\frac{2}{\pi} \int_{0}^{\infty} \frac{\cos p t \sin p z}{p} d p= \begin{cases}1, & z>t, \\
\frac{1}{2}, & z=t, \\
0, & z<t,\end{cases} \\
& \mathcal{I}_{s}(z, t)=\frac{2}{\pi} \int_{0}^{\infty} \frac{\sin p t \sin p z}{p^{2}} d p= \begin{cases}t, & z>t, \\
\frac{(z+t)}{2}, & z=t, \\
z, & z<t .\end{cases}
\end{aligned}
$$

\section{Problem 2}

In this case the inversion integrals can be written in the form

$$
\begin{gathered}
\frac{1}{\theta_{0}} \frac{\partial u_{0}}{\partial z}(z, t)=-\frac{2}{\pi} \int_{0}^{\infty} p\left(U(p, t)-R_{3}(t) \frac{\cos p t}{p^{2}}-S_{3}(t) \frac{\sin p t}{p^{3}}\right. \\
\left.-\frac{h}{p^{2}}\right) \sin p z d p+F_{3}(z, t)+G_{3}(z, t)-h \\
\frac{1}{\theta_{0}} T_{0}(z, t)=\frac{2}{\pi} \int_{0}^{\infty}\left(\begin{array}{c}
\left.T(p, t)-R_{4}(t) \frac{\sin p t}{p^{2}}-\frac{1}{p}\right) \sin p z d p \\
+F_{4}(z, t)+1,
\end{array}\right.
\end{gathered}
$$

where

$$
\begin{aligned}
F_{3}(z, t) & =-R_{3}(t) \mathcal{I}_{c}(z, t) \\
G_{3}(z, t) & =-S_{3}(t) \mathcal{I}_{s}(z, t) \\
F_{4}(z, t) & =R_{4}(t) \mathcal{I}_{s}(z, t)
\end{aligned}
$$

The above analysis allows us to identify the discontinuities in the solutions and their derivatives. We shall denote the jump or discontinuity in a function $F(z, t)$ at $z=t$ by

$$
\left.[F(z, t)]\right|_{z=t}=F(z=t+0, t)-F(z=t-0, t)
$$

and we summarize the discontinuities in the solutions of both problems in table 2. Since the propagation front is at $z=t$ then back in dimensional variables we 


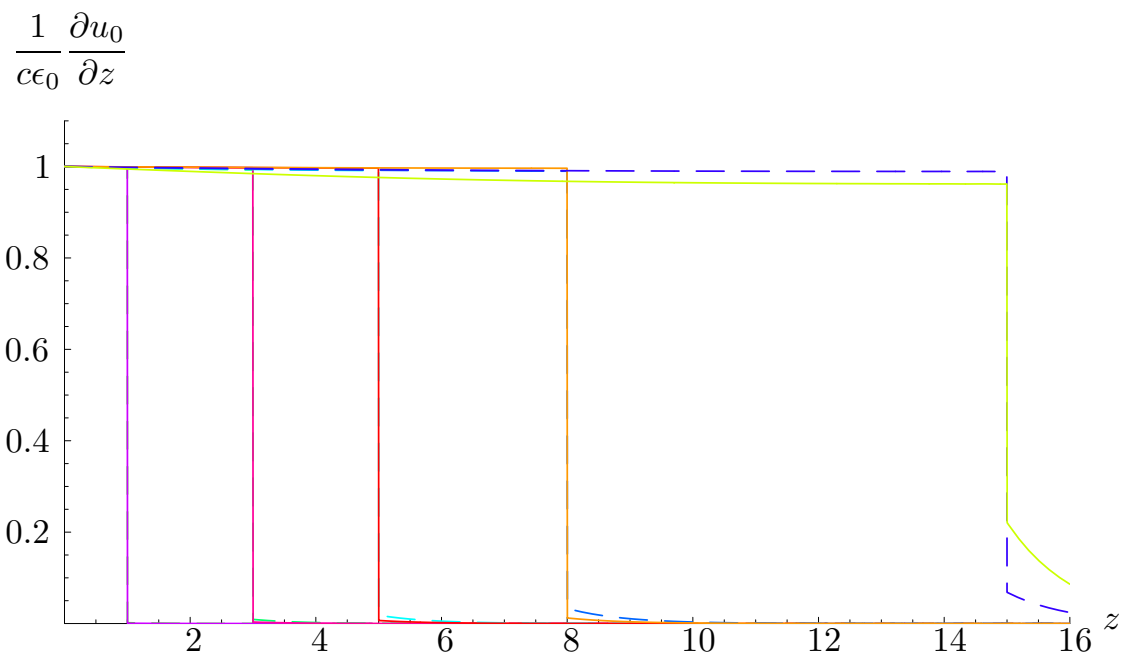

Figure 9: Figure showing the propagation of the homogenized displacement gradient field for Problem 1 at (nondimensional) times $t=1,3,5,8,15$. Note the discontinuity front in the homogenized half-space with $\delta_{*}=0.01132$ (dashed lines) and $\delta=0.0039$ (solid lines). These values correspond to $\phi=0$ (Homogeneous Epoxy half-space) and the minimum coupling parameter when $\phi \approx 0.87$ respectively.

see by referring to (3.1) and (3.2) that the front propagates at speed $v_{*}$ defined in (3.4).

The discontinuities in the solutions of problem 1 exhibit similar behaviour to their homogeneous counterparts in [4] except that here they depend on $\delta_{*}$. In figures 9 and 10 we plot the homogenized displacement gradient and temperature fields respectively, both scaled on $c \epsilon_{0}$. The leading order strain given by (3.13) is of course non-homogenizable [3] since it depends on the microstructure and would possess rapid fluctuations at the interfaces between phases.

The results indicate that the strain boundary condition induces a propagating discontinuity in the displacement gradient and discontinuity in the temperature gradient. The positive dilatation leads to a decrease in absolute temperature in the medium and hence the relative temperature $T_{0}$ is negative. Note that the temperature field has smallest fluctuations for the "optimal" composite (by which we mean minimum thermal coupling) where $\phi=0.87$.

The solutions of problem 2 possess much more complicated behaviour than their homogeneous counterparts in [4]. In particular since $h \neq 0$ in composites, the discontinuities jump up an order so that the displacement gradients and temperature derivatives possess discontinuities which disappear when $h=0$. We illustrate this behaviour in figures 11-13. The stress fields are shown in figure 14 where it is noted once more that there is a discontinuity in the stress field for the "optimal" composite, whereas there is only a discontinuity in its derivative for the homogeneous Epoxy material $(\phi=0)$. 


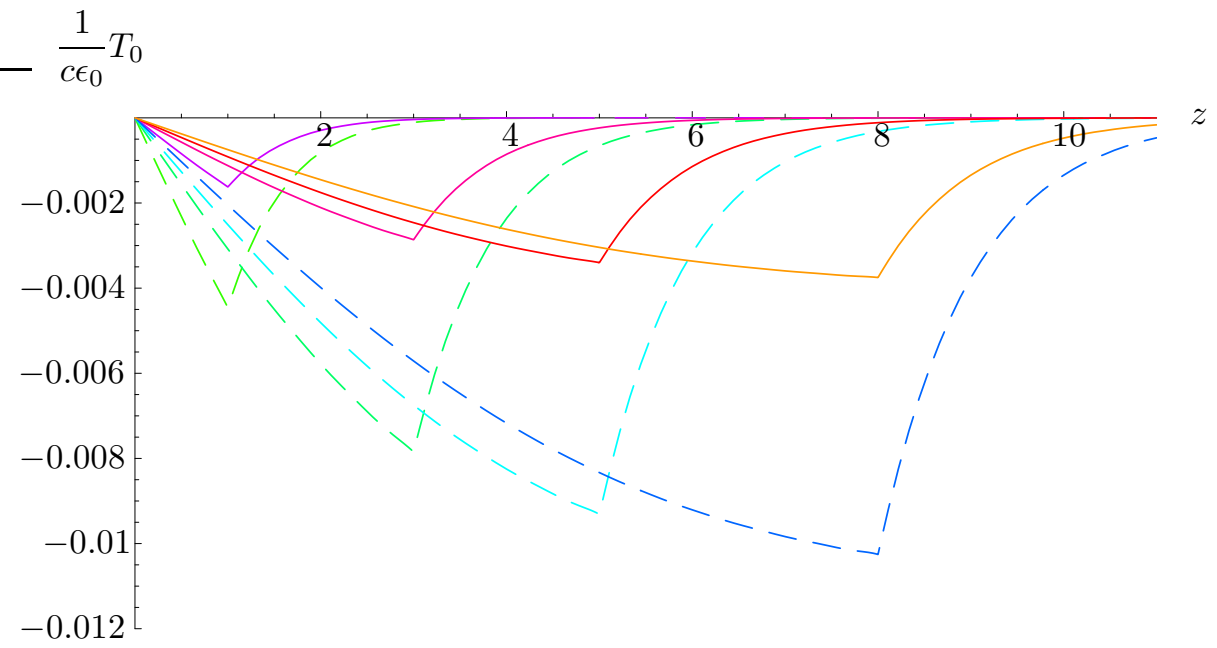

Figure 10: Figure showing the propagation of the temperature field for Problem 1 at (nondimensional) times $t=1,3,5,8$. Note the discontinuity front in the homogenized half-space with $\delta_{*}=0.01132$ (dashed lines) and $\delta=0.0039$ (solid lines). These values correspond to $\phi=0$ (Homogeneous Epoxy half-space) and the minimum coupling parameter when $\phi \approx 0.87$ respectively.

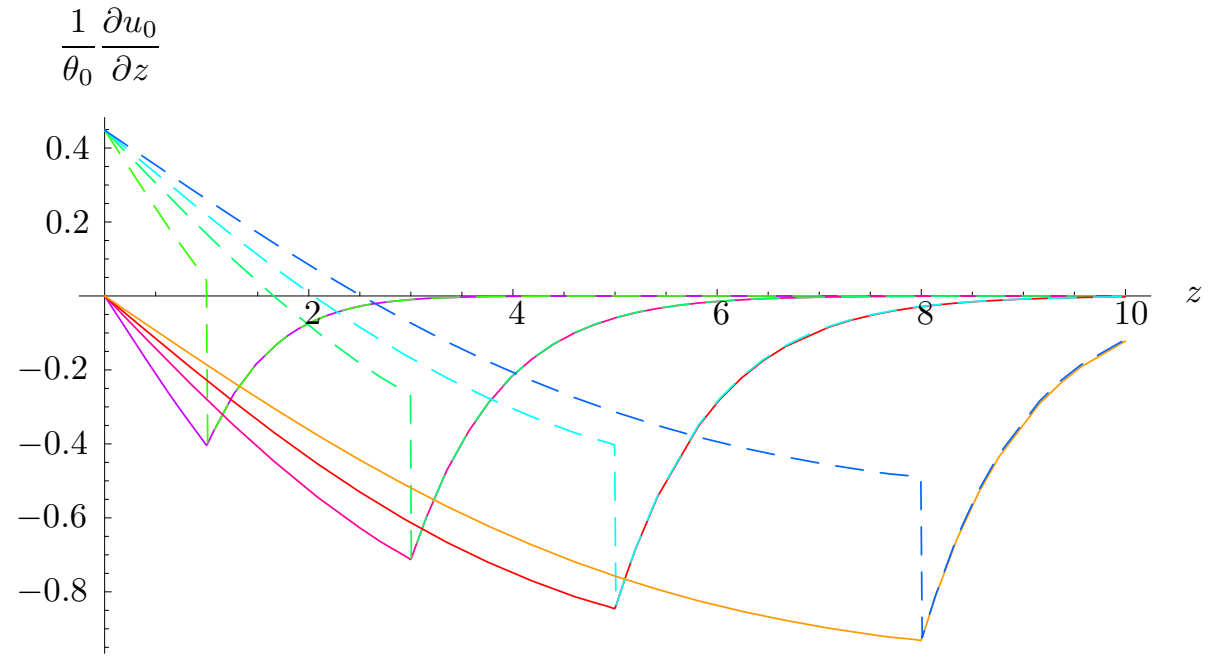

Figure 11: Figure showing the propagation of the homogenized displacement gradient field from Problem 2 at (nondimensional) times $t=1,3,5$ and 8 . Note that the field possesses a discontinuity at $z=t$ except in the cases of $\phi=0$ and $\phi=1$ where the composite is homogeneous. Here we have plotted the cases of the homogenized half-space with $\delta_{*}=0.01132$ (dashed lines) and $\delta_{*}=0.0039$ (solid lines). These values correspond to $\phi=0$ (Homogeneous Epoxy half-space) and the minimum coupling parameter when $\phi \approx 0.87$ respectively. 


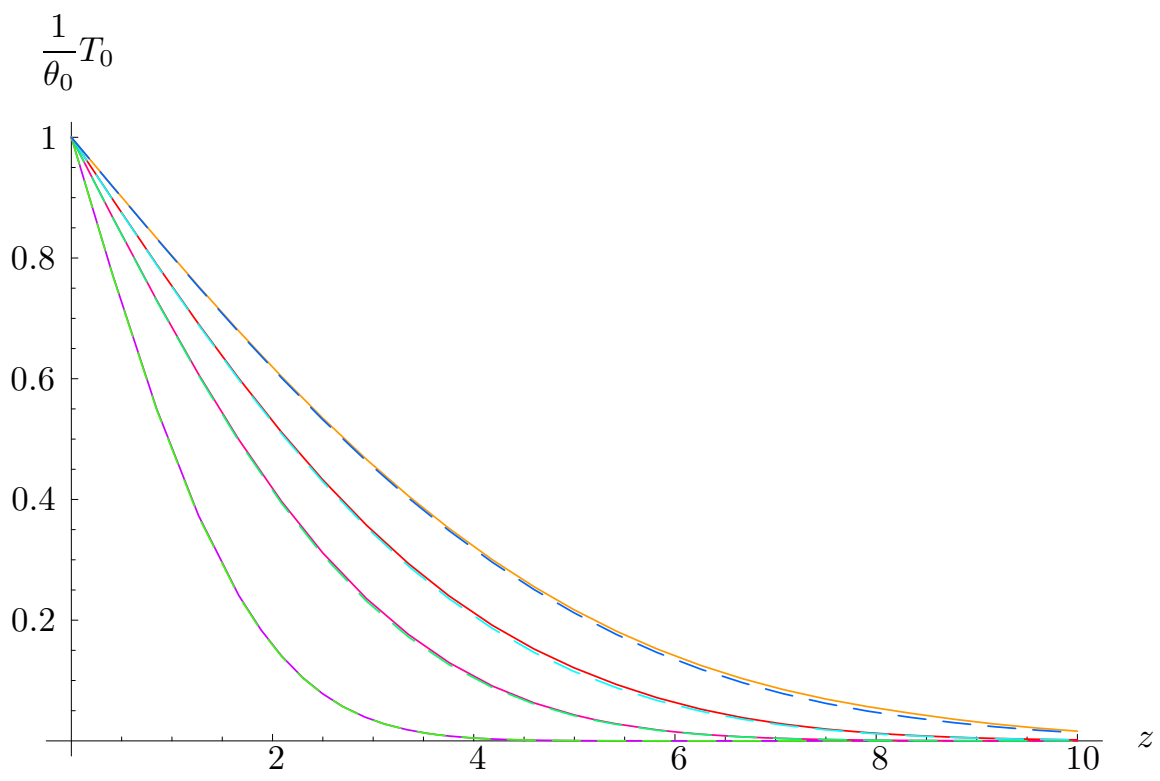

Figure 12: Figure showing the propagation of the homogenized temperature in Problem 2 at (nondimensional) times $t=1,3,5$ and 8 . We have plotted the cases of the homogenized half-space with $\delta_{*}=0.01132$ (dashed lines) and the "optimal" composite with $\delta_{*}=0.0039$ (solid lines). These values correspond to $\phi=0$ (Homogeneous Epoxy half-space) and the minimum coupling parameter when $\phi \approx 0.87$. In the homogenized composite there is a discontinuity in the temperature gradient at $z=t$ but this is difficult to see here. We indicate this in more detail in figure 13. 


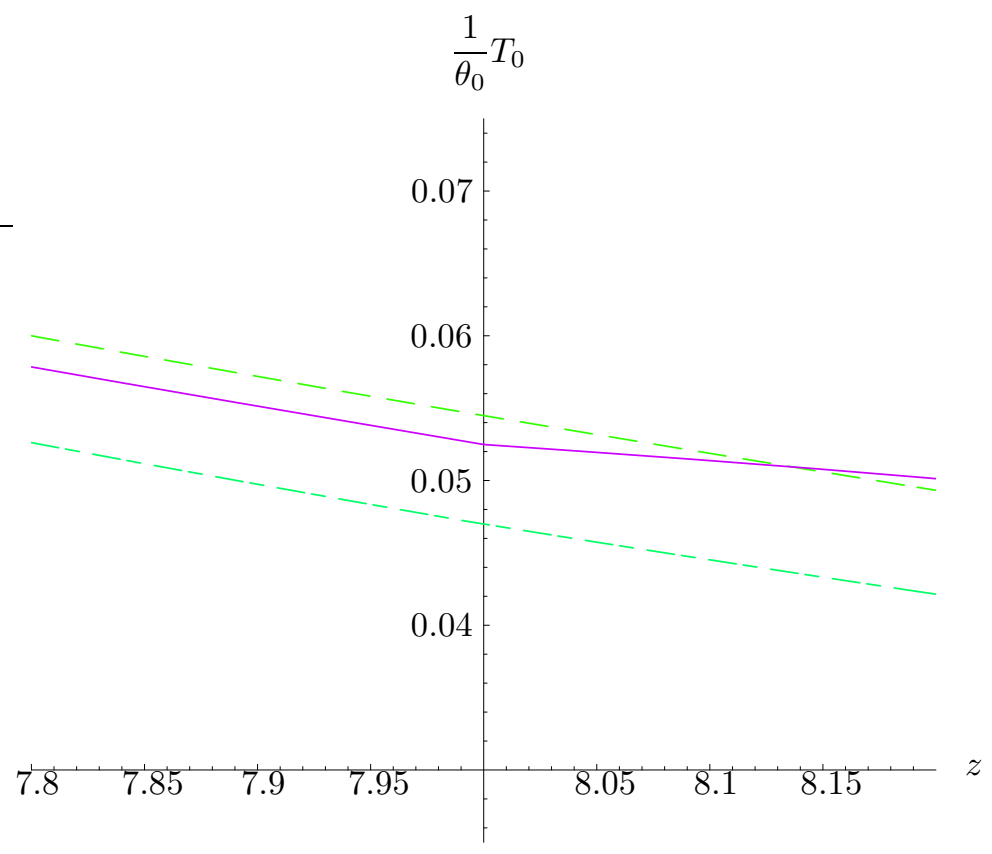

Figure 13: Figure to illustrate the discontinuity in the temperature gradient at $z=t=8$ for three different materials. The dashed line indicates the homogeneous composite with $\phi=0, \delta=0.01132$ and $h=0$ where there is no discontinuity in the derivative. The dash-dotted line indicates the "optimal" composite with $\delta=0.0039$ and $h=-0.48$ where the magnitude of the discontinuity in gradient is very small. The solid line indicates a steel composite with $\delta=0.03$ and $h=-0.6$ where the discontinuity is clearly visible.

$$
\frac{1}{\theta_{0}} \sigma_{0}
$$

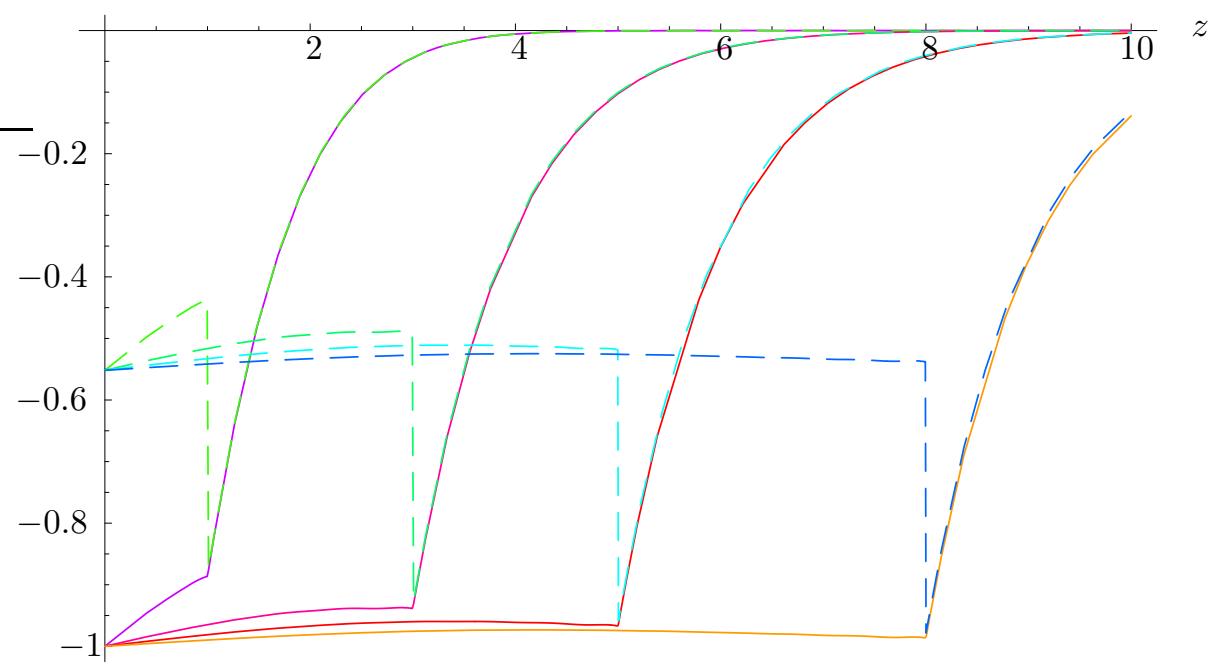

Figure 14: Figure showing the propagation of the stress field in the "optimal" composite with $\delta=0.0039$ (dashed lines) and the homogeneous Epoxy material with $\delta=0.01132$ (solid lines). Note the different discontinuity properties. 


\section{Danilovskaya's Problem}

We can convert the strain and temperature boundary conditions considered above into the more physically meaningful traction and temperature boundary conditions. Using (3.11), the corresponding traction boundary conditions for $t \geq 0$ are

Problem 1

$$
\sigma_{0}(z=0, t)=c \epsilon_{0}, \quad T_{0}(z=0, t)=0 .
$$

Problem 2

$$
\sigma_{0}(z=0, t)=-(1+h) \theta_{0}, \quad T_{0}(z=0, t)=\theta_{0}
$$

and therefore we have solved these problems above.

Let us now consider the following problems, the second of which is the classical Danilovskaya problem [8]:

\section{Problem I}

For $t \geq 0, \quad \sigma_{0}(z=0, t)=\Sigma_{0}, \quad T_{0}(z=0, t)=0$.

The solution to this problem is clearly identical to that obtained via Problem 1 with $c \epsilon_{0}=\Sigma_{0}$.

\section{Problem II}

For $t \geq 0, \quad \sigma_{0}(z=0, t)=0, \quad T_{0}(z=0, t)=\Psi_{0}$.

In order to solve this problem we state it in terms of Problems 1 and 2 above which have already been solved. Consider the form of boundary conditions (3.19)-(3.20) and note from (3.11) that for $\sigma_{0}(z=0, t)=0$ we require

$$
\frac{\partial u_{0}}{\partial z}(z=0, t)=T_{0}(z=0, t)
$$

and thus

$$
f(t)=\left(\frac{1+h}{c}\right) g(t) .
$$

The following strain-temperature boundary conditions for $t \geq 0$ are therefore equivalent to the stress free boundary conditions above:

$$
\begin{aligned}
\frac{\partial u}{\partial x}(z=0, \xi=0, t) & =f(t)=\frac{(1+h)}{c} \Psi_{0}, \\
T_{0}(z=0, t) & =g(t)=\Psi_{0} .
\end{aligned}
$$

Equivalently

$$
\begin{aligned}
\frac{\partial u_{0}}{\partial z}(z=0, t) & =c f(t)-h g(t)=\Psi_{0}, \\
T_{0}(z=0, t) & =g(t)=\Psi_{0} .
\end{aligned}
$$

Thus by linearity, Problem II is equivalent to adding the solution of Problem 1 with

$$
\epsilon_{0}=\frac{(1+h)}{c} \Psi_{0}
$$


to the solution of Problem 2 with

$$
\theta_{0}=\Psi_{0} .
$$

Once more, a summary of the discontinuities in these problems are given in table 2 .

\begin{tabular}{cccc}
\hline Problem & $F(z, t)$ & {$\left.[F(z, t)]\right|_{z=t}$} & {$[\partial F / \partial z]_{z=t}$} \\
\hline 1 & $\frac{1}{c \epsilon_{0}} \frac{\partial u_{0}}{\partial z}$ & $-E(t)$ & $\frac{\delta_{*}}{2}\left(1-\left(1-\frac{\delta_{*}}{4}\right) t\right) E(t)$ \\
& $\frac{1}{c \epsilon_{0}} T_{0}$ & 0 & $\delta_{*} E(t)$ \\
\hline 2 & $\frac{1}{\theta_{0}} \frac{\partial u_{0}}{\partial z}$ & $h E(t)$ & $\left\{1-\frac{h \delta_{*}}{2}\left(1-\left(1-\frac{\delta_{*}}{4}\right) t\right)\right\} E(t)$ \\
& $\frac{1}{\theta_{0}} T_{0}$ & 0 & $-h \delta_{*} E(t)$ \\
\hline I & $\frac{1}{\Sigma_{0}} \frac{\partial u_{0}}{\partial z}$ & $-E(t)$ & $\frac{\delta_{*}}{2}\left(1-\left(1-\frac{\delta_{*}}{4}\right) t\right) E(t)$ \\
& $\frac{1}{\Sigma_{0}} T_{0}$ & 0 & $\delta_{*} E(t)$ \\
\hline II & $\frac{1}{\Psi_{0}} \frac{\partial u_{0}}{\partial z}$ & $-E(t)$ & $\left\{1+\frac{\delta_{*}}{2}\left(1-\left(1-\frac{\delta_{*}}{4}\right) t\right)\right\} E(t)$ \\
& $\frac{1}{\Psi_{0}} T_{0}$ & 0 & $\delta_{*} E(t)$ \\
\hline
\end{tabular}

Table 2: Table exhibiting discontinuities of the functions $F(z, t)$ at $z=t$, for Problems 1, 2, I and II. For conciseness we have written $E(t)=\exp \left(-\delta_{*} t / 2\right)$. Compare these results with the homogeneous case of Boley and Tolins [4], when $h=0$.

\section{Conclusions}

In this article we have considered the problem of the homogenization of a thermoelastic composite half-space, derived the important homogenized parameters and solved some transient fully coupled problems. The homogenization was carried out by the popular and successful asymptotic scheme. Perhaps most importantly we obtained the effective coupling parameter $\delta_{*}$ and a homogenized coupling condition which states when the thermal coupling must be taken into account. Furthermore we showed that within the periodic cell, say $n \epsilon \leq \xi \leq(n+1) \epsilon, n \in \mathbb{Z}, \delta_{*}$ is not bounded below by $\min \{\delta(\xi)\}$, an important result physically, since it means by choosing a composite correctly one can reduce the amount of thermoelastic coupling within the material below that in any of the homogeneous phases. Work is underway to try to establish more formal results regarding the effective coupling parameter and in particular whether lower and upper bounds on $\delta_{*}$ may be established.

We considered some transient boundary value problems analogous to those of Boley and Tolins' [4] homogeneous case. In particular we showed that the discontinuity of the front at $z=t$ can depend on the inhomogeneity parameter $h$ which tends to zero for homogeneous media. 
It is clear that since in some cases the homogenized fields are discontinuous across the front at $z=t$, the process of homogenization could be called into question. We showed formally however that such step problems can be considered as the limit of a homogenizable problem and as such provide us with qualitative information about the behaviour of real physical problems with slightly smoother boundary conditions (which still vary rapidly on the macroscale), where thermal coupling still needs to be accounted for.

The problems considered are instructive in terms of homogenization and what may be concluded about a homogenized thermoelastic material. As is well known, stress and temperature fields can be homogenized but strain fields cannot. Thus no meaning can be attached to the concept of the homogenized strain field or temperature flux in the composite half-space.

It is felt that the above approach is an improvement over the theory proposed by Baczynski [2] since here we solve the fully coupled problem using a well established theory of homogenization, an effective coupling parameter is defined, a coupling condition is established and the magnitude of the discontinuities at the front $z=t$ are provided. These are all improvements over Baczynski's work and, as far as the author is aware, are new results.

It would be useful to obtain the results for an impulsive boundary condition in the composite case since this corresponds to the Green's function for the material. Secondly, it would be useful to consider thermoelastic composite slabs where end effects are important and the propagation behaviour of a front within such a slab.

A convenient integral form for the solutions for small times is obtained by understanding the behaviour of the solutions as $p \rightarrow \infty$. In this limit it can be shown that

$$
\begin{aligned}
& \alpha \sim\left(p^{2}-\delta_{*}\right)-\frac{\delta_{*}\left(\delta_{*}-1\right)}{p^{2}}+O\left(\frac{1}{p^{4}}\right), \\
& \beta \sim \frac{\delta_{*}}{2}+\frac{\delta_{*}\left(\delta_{*}-1\right)}{2 p^{2}}+O\left(\frac{1}{p^{4}}\right), \\
& \gamma \sim p-\frac{\delta_{*}\left(\delta_{*}-4\right)}{8 p}+O\left(\frac{1}{p^{4}}\right) .
\end{aligned}
$$

\section{Problem 1}

Therefore we find that as $p \rightarrow \infty$,

$$
\begin{aligned}
& \frac{U(p, t)}{c \epsilon_{0}} \sim-\frac{1}{p^{2}}+R_{1}(t) \frac{\cos p t}{p^{2}}+S_{1}(t) \frac{\sin p t}{p^{3}}+O\left(\frac{1}{p^{4}}\right), \\
& \frac{V(p, t)}{c \epsilon_{0}} \sim R_{2}(t) \frac{\sin p t}{p^{2}}+O\left(\frac{1}{p^{3}}\right),
\end{aligned}
$$

where

$$
\begin{gathered}
R_{1}(t)=\exp \left(\frac{-\delta_{*} t}{2}\right), \quad R_{2}(t)=-\delta_{*} \exp \left(\frac{-\delta_{*} t}{2}\right), \\
S_{1}(t)=\frac{\delta_{*}}{2} \exp \left(\frac{-\delta_{*} t}{2}\right)\left(1-\left(1-\frac{\delta_{*}}{4}\right) t\right) .
\end{gathered}
$$




\section{Problem 2}

In this case we find that as $p \rightarrow \infty$,

$$
\begin{aligned}
& \frac{U(p, t)}{\theta_{0}} \sim \frac{h}{p^{2}}+R_{3}(t) \frac{\cos p t}{p^{2}}+S_{3}(t) \frac{\sin p t}{p^{3}}+O\left(\frac{1}{p^{4}}\right), \\
& \frac{V(p, t)}{\theta_{0}} \sim \frac{1}{p}+R_{4}(t) \frac{\sin p t}{p^{2}}+O\left(\frac{1}{p^{3}}\right),
\end{aligned}
$$

where

$$
\begin{array}{r}
R_{3}(t)=-h \exp \left(\frac{-\delta_{*} t}{2}\right), \quad R_{4}(t)=h \delta_{*} \exp \left(\frac{-\delta_{*} t}{2}\right), \\
S_{3}(t)=\exp \left(\frac{-\delta_{*} t}{2}\right)\left\{1-\frac{h \delta_{*}}{2}\left(1-\left(1-\frac{\delta_{*}}{4}\right) t\right)\right\} .
\end{array}
$$

These forms are then subtracted from the integrands in the inversion integ-

rals in order to ensure better convergence of the integrals as we see in section 6.

\section{References}

[1] J.D. Achenbach. Approximate transient solutions for the coupled equations of thermoelasticity. Journal of the Acoustical Society of America, 36(1):10-18, 1963.

[2] Z.F. BACZynski. Dynamic thermoelastic processes in microperiodic composites. Journal of Thermal Stresses, 26(1):55-66, 2003.

[3] N. Bakhvalov and G. Panasenko. Homogenization: Averaging Processes in Periodic Media. Kluwer, 1989.

[4] B.A. Boley and I.S. Tolins. Transient coupled thermoelastic boundary value problems in the half-space. Journal of Applied Mechanics, 29:637646, 1962.

[5] B.A. Boley And J. Weiner. Theory of Thermal Stresses. John Wiley and Sons, 1960.

[6] D. Bouzit And C. Pierre. An experimental investigation of vibration localization in disordered multispan beams. J. Sound Vibr., 187:649-669, 1995 .

[7] B. Budiansky. Thermal and thermoelastic properties of isotropic composites. J. Composite Materials, 4:286-295, 1970.

[8] V.I. Danilovskaya. Thermal stresses in an elastic halfspace arising after a sudden heating of its boundary (in Russian). Prikladnaya Matematika $i$ Mechanika, 14:316-318, 1950.

[9] G.A. FrancFort. Homogenization and linear thermoelasticity. SIAM Journal on Mathematical Analysis, 14(4):696-708, 1983. 
[10] E.H. Kerner. The elastic and thermo-elastic properties of composite media. Proc. Phys. Soc. B, 69:808-813, 1956.

[11] N. Laws. On the thermostatics of composite materials. Journal of the Mechanics and Physics of Solids, 21:9-17, 1973.

[12] W.J. Parnell and I.D. Abrahams. Dynamic homogenization in periodic fibre reinforced media. I. Quasi-static limit for SH waves. Submitted to Wave Motion, 2005.

[13] W.J. Parnell and I.D. Abrahams. An Introduction to Homogenization in Continuum Mechanics. Cambridge University Press, 2005.

[14] J.N. RedDy. Mechanics of laminated Composite Plates and Shells: Theory and Analysis. CRC Press, 2003.

[15] F. Santosa and M. Vogelius. First order corrections to the homogenized eigenvalues of a periodic composite medium. SIAM J. Appl. Math., 53:1636-1668, 1993.

[16] G. Solaroli, Z. Gu, A. Baz, and M. Ruzzene. Wave propagation in periodic stiffened shells: Spectral finite element modelling and experiments. J. Vibr. Control, 9:1057-1081, 2003.

[17] J.D. Whitcomb And J. Noh. Concise derivation of formulas for 3d sublaminate homogenization. J. Composite Materials, 34(6), 2000. 